\title{
Acerca de la articulación de la lectura epistémica del Idealismo Trascendental y una interpretación realista del concepto crítico de cosa en sí
}

\author{
On the epistemic reading of Transcendental Idealism \\ and a realistic interpretation of the critical concept \\ the thing in itself \\ ILEANA P. BEADE \\ Universidad Nacional de Rosario (Argentina) \\ Consejo Nacional de Investigaciones Científicas y Técnicas (CONICET)
}

\section{RESUMEN}

En este escrito proponemos examinar en qué sentido la interpretación epistémica de la distinción crítica fenómeno/cosa en sí resulta compatible con la interpretación de la cosa en sí como entidad real. Atendiendo a este objetivo, se intentará mostrar que dicha interpretación epistémica, lejos de reducir la cosa en sí a un mero concepto heurístico, conduce a una consideración de la misma como entidad efectivamente existente (más allá de las dificultades que obviamente supone la aplicación de las categorías de realidad y existencia a aquello que, por definición, jamás puede ser dado como objeto de experiencia). 


\begin{abstract}
In this paper I shall analyze in what sense the Epistemic Interpretation of the critical distinction between appearances and things in themselves is compatible with an interpretation of the thing in itself as a real entity. I will try to show that the epistemic interpretation, far from reducing the thing in itself to a mere heuristic concept, leads to a consideration of the thing in itself as an existing entity (notwithstanding the difficulties which are obviously involved in the application of the categories of reality and existence to what can never be given as an object of experience).
\end{abstract}

KEYWORDS

APPEARANCE, THING IN ITSELF, PERSPECTIVE, REALITY

\title{
I. INTRODUCCIÓN
}

Si BIEN LA DOCTRINA KANTIANA rechaza la posibilidad de alcanzar un conocimiento de las cosas en sí mismas, en numerosos pasajes de la Crítica de la razón pura -y de otros textos pertenecientes al período crítico- hallamos importantes observaciones referidas a la cosa en sí, caracterizada, en ocasiones, como correlato necesario del fenómeno, ${ }^{1}$ como fundamento suprasensible de aquél, ${ }^{2}$ o como entidad que afecta nuestra sensibilidad dando así origen a las representaciones empíricas. ${ }^{3}$ Las objeciones tradicionalmente formuladas al concepto de cosa en sí suelen remitir a esta aparente tensión, considerada, por muchos, como el auténtico «talón de Aquiles» de la epistemología crítica. Dichas objeciones resultan, por otra parte, especialmente relevantes y significativas, si se tiene en cuenta que la interpretación del concepto de cosa en sí ha sido -y continúa siendo- decisiva para la interpretación de la doctrina del Idealismo trascendental en su conjunto, ${ }^{4}$ tal como evidencian las más recientes

$1 C f$. Ak. IV, 354. Citamos la paginación correspondiente a la edición académica de las obras kantianas: Kant's gesammelte Schriften, hrsg. von der Königlich Preussischen, bzw. der Deutschen Akademie der Wissenschaften, Berlín et alia, 1902 ss., I-IX (a esta edición aludimos bajo la abreviatura Ak., seguida del número de tomo, indicado en números romanos). En el caso de la Crítica de la razón pura [Kritik der reinen Vernunft, 1781/1787], nos referimos a la primera y a la segunda edición bajo las abreviaturas A y B, según el uso convencional. Citamos la traducción castellana: I. Kant, Crítica de la razón pura, tr. Mario Caimi. Buenos Aires: Colihue, 2007.

2 Cf. A 277/B 333; A 358; A 379ss; A 538/B 566; A 613/B 641; Ak. VIII, 215.

3 Cf. A 494/B 522.

4 En relación con la importancia fundamental que el análisis de los conceptos de fenómeno y cosa en sí cobra para la interpretación de la doctrina kantiana, cabe señalar que tanto la distinción entre fenómenos y cosas en sí como la tesis que establece la imposibilidad de un conocimiento de estas últimas, constituyen principios fundamentales del Idealismo Trascendental 
discusiones entre los partidarios de la interpretación epistémica de dicha doctrina (sustentada en una lectura epistémica de la distinción fenómeno / cosa en sí) y aquellos que suscriben, por el contrario, una interpretación ontológica. ${ }^{5}$ Tratándose de un concepto cuyo análisis exige el tratamiento de diversas problemáticas específicas, en esta oportunidad nos limitaremos considerar en qué sentido la interpretación epistémica de la distinción fenómeno /cosa en sí

(IT); tal como puede ser constatado a partir del análisis de las dos definiciones del IT formuladas en la Crítica de la razón pura (cf. A 490-491/B 518-519; A 369).

5 La interpretación epistemológica -impulsada por G. Prauss y, más recientemente, por $\mathrm{H}$. Allison- sostiene, en términos generales, que la distinción en cuestión remite a dos maneras de considerar un objeto único (a saber, el objeto empírico, dado en la experiencia), mientras que los adeptos de la interpretación ontológica suelen rechazar esta posición, alegando que el fenómeno y la cosa en sí resultan irreductibles, habida cuenta del sentido eminentemente ontológico que el concepto de cosa en sí asume en el marco de la filosofía crítica. Cabe señalar, sin embargo, que si bien el debate entre ambas líneas de interpretación ha sido frecuentemente caracterizado a través de la oposición entre las llamadas «interpretación de los dos aspectos» y la «interpretación de los dos mundos», una breve reconstrucción de las principales discusiones que configuran el estado actual de los estudios kantianos permite constatar que dicha oposición no alcanza ya a reflejar la complejidad que ha cobrado la discusión durante las últimas dos décadas. En efecto, buena parte de las actualmente denominadas interpretaciones ontológicas incorporan ciertos aspectos o resultados parciales de la interpretación epistémica, rechazando así la interpretación dualista radical del Idealismo kantiano, esto es, aquella que lo interpreta como una doctrina que postula la existencia de dos mundos ontológicamente diversos e irreductibles: el sensible y el inteligible ( $c f$. D. Schulting, «Kant's Idealism: The Current Debate», en D. Schulting y J. Verburgt, (eds.), Kant's Idealism. New Interpretations of a Controversial Doctrine. Springer, 2010, pp. 1-35; D. Heidemann, «Appearance,Thing-in-Iself, and the Problem of the Skeptical Hypothesis», en D. Schulting y J. Verburgt, (eds.), Kant's Idealism. New Interpretations of a Controversial Doctrine. Springer, 2010, p. 201). Para un análisis detallado de estas diversas líneas de interpretación, véase: L. Allais, «Kant's one world: Interpreting Transcendental Idealism», British Journal for the History of Philosophy, 12, 4 (2004), pp. 655ss.; C. Onof, «Thinking the In-itself, and Its Relation to Appearances», en D. Schulting y J. Verburgt (eds.), Kant's Idealism. New Interpretation of a Controversial Doctrine. Springer, 2010, pp. 212ss.; K. Ameriks, «Kantian Idealism today», en K. Ameriks, Interpreting Kant's Critiques. Oxford: Clarendon Press, 2003, pp. 98-99. Para el análisis de los principios y conceptos básicos sobre los que se sustentan las recientes interpretaciones ontológicas del IT, remitimos a los textos de K. Ameriks: "Kant's Idealism on a Moderate Interpretation», en D. Schulting y J. Verburgt (eds.), Kant's idealism. New Interpretation of a Controversial Doctrine. Springer, 2010, pp. 29-53; «The critique of metaphysics: Kant and traditional Ontology», en P. Guyer (ed.), The Cambridge Companion to Kant. Cambridge: Cambridge University Press, 1992, pp. 258-272; «The critique of metaphysics. The structure and fate of Kant's Dialectic», en P. Guyer (ed.), The Cambridge Companion to Kant and Modern Philosophy. Cambridge: Cambridge University Press, 2007, pp. 282-283. A modo de ejemplo paradigmático de la lectura epistémica del IT, vid. H. Allison, Idealism and Freedom. Essays on Kant's Theoretical and Practical Philosophy. Cambridge: Cambridge University Press, 1996, pp. 20ss. 
-que aquí suscribimos- permite ser articulada con una interpretación realista de la cosa en sí. En otras palabras: intentaremos mostrar que la interpretación epistémica, lejos de reducir la cosa en sía un mero concepto heurístico, resulta compatible con una interpretación de la cosa en sí como entidad efectivamente real o existente (más allá de las dificultades que obviamente supone la aplicación de las categorías de realidad y existencia a aquello que, por definición, jamás puede ser dado como objeto de experiencia ${ }^{6}$ ).

Atendiendo a este objetivo, en la primer sección ofrecemos una breve reconstrucción de los lineamientos básicos de la lectura epistémica de la distinción fenómeno / cosa en sí, a partir de un análisis de los principales pasajes en los que Kant se refiere explícitamente al sentido epistemológico que ambos términos cobran en el marco de la investigación trascendental. En la segunda sección -y sobre la base de las observaciones realizadas en la sección primera- procuraremos indicar las razones por las que consideramos que la lectura epistémica permite establecer el carácter real de las entidades a las que Kant caracterizó bajo el concepto de cosa en sí, señalando asimismo los aspectos conceptuales básicos sobre los que se apoyar la interpretación realista de la cosa en sí.

\section{LA OPOSICIÓN FENÓMENO / COSA EN SÍ COMO DISTINCIÓN EPISTÉMICA}

La distinción fenómeno / cosa en sí aparece, en el marco de la investigación crítica, como resultado de las investigaciones llevadas a cabo en la Estética trascendental, sección de la Crítica de la razón pura en la que se expone la concepción crítica del espacio y el tiempo como formas a priori de la sensibilidad. Si el espacio y el tiempo no son reales en sí, sino meras condiciones subjetivas de nuestra facultad de representación sensible, ello conduce a la conclusión de que las determinaciones espacio-temporales de los objetos que se nos presentan en la experiencia no corresponden a los objetos tal como son en sí mismos, sino únicamente a los objetos tal como aparecen ante nuestra sensibilidad, es decir,

6 A tales dificultades nos hemos referido en detalle en trabajos previos. $C f$. I. Beade, «La cosa en sí y su rol en la constitución de la objetividad. Una lectura crítica de la interpretación de Ch. Onof», en M. Damiani (ed.), Actas del I Simposio de Filosofía Moderna, Rosario: Centro de Estudios de Filosofía Moderna, 2011, en prensa; I. Beade, «Acerca del concepto kantiano de fenómeno y su relación con el concepto crítico de cosa en sí», en D. López, M. Yuan y C. Lammertyn (eds.), Experiencia y concepto. Intensidades clásicas y tensiones contemporáneas. Santa Fe: Ediciones de la Universidad Nacional del Litoral, 2011, pp. 254-260; I. Beade, "Acerca de la cosa en sí como causa de la afección sensible", Signos Filosóficos, XII, 23 (2010), pp. 9-37; I. Beade, «Realidad y perspectiva. Consideraciones acerca del concepto de cosa en sí en el marco de la Gnoseología crítica», en D. López y S. Di Sanza (eds.), El idealismo alemán. Autores y textos. Santa Fe: Ediciones de la Universidad Nacional del Litoral, en prensa; I. Beade, «Consideraciones acerca del concepto kantiano de objeto trascendental», Tópicos, 22 (2009), pp. 85-120. 
corresponden únicamente a las cosas en tanto fenómenos. En tal sentido señala Kant, en las conclusiones subsiguientes a las Exposiciones del espacio, que no podemos hacer de las condiciones de la sensibilidad condiciones de las cosas en general (cf. A 27/ B 43). No hay, en efecto, razón alguna para suponer que una condición subjetiva (inherente al modo en que opera nuestra facultad de representación sensible) constituya, a la vez, una determinación inherente a las cosas en sí mismas, es decir, a las cosas tal como éstas son independientemente de toda relación con nuestra facultad de representación. ${ }^{7}$ Así concluye Kant que «toda nuestra intuición no es nada más que la representación del fenómeno; que las cosas que intuimos no son, en sí mismas, como se nos aparecen; ni sus relaciones están constituidas, en sí mismas, como se nos aparecen» (A 42/B 59). La concepción crítica del objeto dado en la experiencia como puro fenómeno implica, así, una referencia tácita al concepto de cosa en sí, pues -como señala el filósofo- sería absurdo que hubiese fenómenos sin que hubiese algo que apareciese. ${ }^{8}$

Ahora bien, la distinción fenómeno / cosa en sí puede ser establecida según dos sentidos diversos, a saber: como distinción empírica, o como distinción trascendental. En el primer caso, se trata de aquella distinción que suele establecerse, en el nivel empírico, entre el objeto externo (al que consideramos como algo independiente de nuestra facultad de representación, es decir, como algo en sí) y la representación mental, correspondiente a dicho objeto. A esta concepción empírica del objeto externo como algo en sí-esto es, como entidad independiente de la conciencia- se refiere Kant cuando afirma que el objeto dado en la experiencia vale, en el nivel empírico, como cosa en sí: «aquello que originariamente es, ello mismo, sólo fenómeno, por ejemplo, una rosa, vale, en sentido empírico, por una cosa en sí misma» (A 30/B 45). Al asumir, en cambio, la perspectiva propia de la reflexión trascendental, el filósofo crítico revela que ese objeto externo -considerado, en el nivel empírico, como una entidad independiente de la conciencia- no constituye, bajo esta nueva consideración, algo real en sí, pues sus determinaciones sensibles -i.e. espacio-temporales- se

7 Para un análisis de las discusiones en torno a la tesis crítica que establece el carácter suprasensible de las cosas en sí, véase: L. Falkenstein, «Kant's Argument for the Non-Spatiotemporality of Things in Themselves», Kant-Studien, 80 (1989), pp. 265-283; Ch. Huenemann, «A Note on the Argument fot the Non-Spatiotemporality of Things in Themselves. A response to Lorne Falkenstein», Kant-Studien, 83 (1993), pp. 381-383; F. X. Chenet, «Que sont donc l'espace et le temps? Les hypothèses considérées par Kant et la lancinante objection de la «troisième possibilité»» Kant-Studien, 84 (1993), pp. 129-153.

$8 C f$. B XXVI-XXVII. El término Erscheinung (fenómeno) proviene del verbo erscheinen (aparecer); de allí que el concepto de fenómeno remita necesariamente a algo que aparece, algo que no constituye, en sí, una entidad fenoménica, y que es denominado, en el marco de la filosofía crítica, bajo el concepto de cosa en sí. 
fundan en la constitución peculiar de nuestra sensibilidad. En el nivel propio del análisis trascendental, puede afirmarse, pues, que el objeto dado en la experiencia es un fenómeno, i.e. constituye un objeto formalmente configurado a partir de una serie de condiciones subjetivas de la sensibilidad (en tal sentido sostienen Kant que tal objeto constituye una representación y no algo en sí). ${ }^{9}$

Como ha señalado Prauss, la diferencia fundamental entre la distinción empírica y la distinción trascendental entre fenómeno y cosa en sí consiste en que, en el caso de la distinción empírica, se establece una oposición entre dos «entidades» diversas (la representación mental y el objeto externo), mientras que, en el caso de la distinción trascendental, se contraponen, en cambio, dos maneras de considerar una única entidad..$^{10}$ En efecto, la distinción trascendental supone una diferenciación entre dos perspectivas bajo las cuales ha de ser considerado el objeto empírico: al considerarlo en su dependencia respecto de las condiciones subjetivas de la representación, lo consideramos comofenómeno; al considerarlo, en cambio, más allá de toda relación con dichas condiciones, lo consideramos como algo en sí. ${ }^{11}$ La diferenciación entre un sentido empírico y un sentido trascendental de la distinción fenómeno / cosa en sí permite advertir -según observa Prauss- el equívoco en el cual se basa, en última instancia, la interpretación ontológica de dicha distinción: dado que el fenómeno y la cosa en sí en sentido empírico constituyen dos realidades diversas (a saber: la representación mental y el objeto externo), podría pensarse que la distinción trascendental entre el fenómeno y la cosa en sí alude, del mismo modo, a dos entidades diversas

9 Esto no implica, sin embargo, que el objeto físico sea reducido, en la doctrina crítica, a una representación puramente subjetiva: el fenómeno constituye, por el contrario, una representación de carácter objetivo, es decir, válida para todo sujeto en general y, posee, en cuanto tal, realidad en sentido empírico (cf. A 28/B 44).

10 «Empirische Erscheinungen und empirische Dinge an sich sind numerisch-existenziell different [...] so sind doch Erscheinungen und Dinge an sich im philosophischen Sinne numerisch-existenziell identisch» (G. Prauss, Kant und das Problem der Dinge an sich, Bonn: Bouvier Verlag, 1989, p. 52). A partir de esta observación el autor concluye que la distinción trascendental fenómeno / cosa en sí no ha de ser interpretada en sentido ontológico-metafísico, sino en sentido estrictamente epistemológico. Allison coincide con Prauss respecto de este punto: «Thus, unlike the empirical version on the distinction, when these terms are taken in their transcendental sense, they refer not to two classes of entity, one «in the mind» and the other independent of it, but two points of view or perspectives from which one and the same entity (the empirical object) can be considered» (H. Allison, «Kant's Refutation of Realism», Dialéctica, 30, 2/3 (1976) pp. 287-288).

11 Como señala Allison, si bien en ciertos pasajes Kant tiende a identificar el objeto empírico con el fenómeno mismo, son en rigor los objetos empíricos (dados en la experiencia) aquellos que han de ser considerados desde dos perspectivas diversas y complementarias, a saber: como fenómenos o como cosas en sí ( $c f$. H. Allison, Kant's Transcendental Idealism, New Haven: Yale University Press, 2004, p. 61). 
independientes. ${ }^{12}$ Sin embargo, numerosos pasajes de los textos críticos permiten constatar que la distinción trascendental que Kant establece entre fenómeno y cosa en sí es de carácter epistemológico, por cuanto alude -reiteramos- a dos perspectivas bajo las cuales ha de ser considerado el objeto de experiencia en el marco de la reflexión filosófica. ${ }^{13}$ La primera de estas perspectivas (que remite al objeto como fenómeno) se presenta -según indicamos- como resultado de la investigación desarrollada en la Estética trascendental (una vez que ha sido determinado el carácter ideal de las representaciones del espacio y del tiempo se arriba, en efecto, a la concepción del objeto dado en la experiencia como puro fenómeno); la segunda perspectiva se presenta, a su vez, como resultado de la primera, pues la concepción del objeto como fenómeno exige ser contrapuesta a la consideración del objeto como cosa en sí:

Se sigue también, de manera natural, del concepto de un fenómeno en general: que a éste debe corresponderle algo que no es, en sí, fenómeno, porque un fenómeno no puede ser nada en sí mismo y fuera de nuestro modo de representación, y por tanto, si no ha de resultar un perpetuo círculo, la palabra fenómeno indica ya una referencia a algo cuya representación inmediata es, ciertamente, sensible, pero que en sí mismo, sin esta constitución de nuestra sensibilidad (en la cual tiene su fundamento la forma de nuestra intuición), debe ser algo, es decir, un objeto independiente de la sensibilidad. (A 251-252).

$12 C f$. G. Prauss, op. cit., pp. 52ss.

13 Adickes observa, a propósito de esta cuestión: «Trotzdem aber bleibt es dabei, dass ein und derselbe Gegenstand zugleich Ding an sich und Erscheinung ist, das Ding an sich in ihm zwar unerkennbar, aber doch eben in ihm erscheinend, in ihm manifestierend» (E. Adickes, Kant und das Ding an Sich. Berlín: Panverlag Rolf Heise, 1924, p. 20). Adickes señala que la cosa en sí no es sino el fenómeno mismo, en tanto se lo libera de toda referencia a las condiciones subjetivas de la representación (cf. op. cit., pp. 27, 122, 125). Asumiendo una posición similar acerca de esta cuestión, señala Paton: «Kant never ceases to hold that the phenomenal objects we know are only appearances of unknown things-in-themselves. On this view there are not two objects, but only one considered from different posits of view: the thing as it is in itself, and the same thing as it appears to us» (H. J. Paton, Kant's Metaphysic of Experience. A Commentary on the First Half of the Kritik der reinen Vernunft Londres: Georg Allen \&. Unwin, 1970, vol. I, p. 422). Westphal coincide con Adickes y Paton: «The thing in itself is the same thing as the appearance. These are not two objects, but one, which may be considered in two ways. This is most clearly expressed by referring to objects as they are in themselves and as they (i. e., those same things) appear to us [...]. Of course the identity of thing in itself and appearance is interesting only because of the difference it includes. This difference Kant locates in diverse modes of knowing the same object» (M. Westphal, «In Defense of the Thing in Itself», Kant-Studien, 59 (1968), pp. 120-121). 
Si consideramos, como es justo, los objetos de los sentidos como meros fenómenos, por lo mismo admitimos al mismo tiempo, que en el fundamento de ellos yace una cosa en sí misma, aunque a ésta no la conozcamos tal como está constituida en sí misma, sino que conozcamos sólo su fenómeno [...]. Por consiguiente, precisamente porque admite fenómenos, el entendimiento acepta también la existencia de cosas en sí mismas [das Dasein von Dingen an sich selbst]. (Ak. IV, 314-315). ${ }^{14}$

Al caracterizar a los objetos dados en la experiencia como fenómenos, asumimos, pues, tácitamente que éstos re-presentan un «objeto independiente de la sensibilidad» (objeto al que alude, precisamente, el concepto de cosa en sí). Ahora bien, esta correlación entre las nociones de fenómeno y cosa en sí no ha de entenderse -y este es un punto central para nuestro análisis- como una correlación entre dos entidades ontológicamente diversas e independientes, sino como una correlación entre dos dimensiones propias de un objeto único existente. En efecto, Si Kant afirma que al establecer el carácter fenoménico de los objetos dados en la experiencia admite, al mismo tiempo, la existencia de la cosa en sí, es porque considera que la existencia de lo en sí se halla comprendida en la existencia misma del fenómeno sensible (el cual no es, en rigor, sino la cosa misma que aparece ante nosotros, permaneciendo, sin embargo, desconocida en sí, a causa de las condiciones formales que necesariamente imponemos a las cosas en tanto las representamos).

Fenómeno y cosa en sí no constituyen, así pues, entidades realmente diversas -i.e. ontológicamente independientes-, sino que se trata aquí de nociones que remiten a dos modalidades de existencia constitutivas de una única entidad: el objeto empírico (que será considerado, en el marco de la reflexión trascendental, bajos perspectivas diversas). ${ }^{15}$ En tal sentido señala Kant, en el Prólogo a la segunda edición de la Crítica de la razón pura, que la doctrina crítica nos enseña «a tomar al objeto en una doble significación, a saber, como fenómeno o como cosa en sí misma» [das Objekt in zweierlei Bedeutung nehmen lehrt, nämlich als Erscheinung, oder als Ding an sich] (B XXVII), observación denota el sentido originariamente epistemológico que el filósofo atribuye a las nociones de fenómeno y de cosa en sí.

14 La cita corresponde al texto Prolegomena zu einer jeden künftigen Metaphysik, die als Wissenschaft wird auftreten können (1783). Citamos la versión castellana: Kant, I., Prolegómenos a toda metafísica que haya de poder presentarse como ciencia, tr. M. Caimi. Madrid: Istmo, 1999.

15 Podría afirmase, así, que ambas nociones poseen -pese a su sentido originariamente epistémico- cierto sentido ontológico, siempre y cuando no se concluya a partir de ello que la distinción en cuestión supone la adhesión a un dualismo ontológico (esto es: conduce a una concepción del fenómeno y de la cosa en sí como dos entidades ontológicamente independientes). 
Ello no significa, sin embargo, que la distinción epistémica entre el objeto considerado como fenómeno y el objeto considerado como algo en sí carezca de consecuencias ontológicas o metafísicas. Muy por el contrario, al establecer el carácter ideal del espacio y el tiempo, la epistemología crítica establece asimismo, y como consecuencia de ello, que las determinaciones espaciotemporales propias de los objetos dados en la experiencia corresponden a éstos sólo en la medida en que son representados (no corresponden, por consiguiente, a los objetos en sí). De allí se deduce, a su vez -si bien de manera puramente negativa-, el carácter no sensible (o suprasensible) de las cosas en sí, tesis de indudable connotación metafísica. En todo caso, interesa señalar, por el momento, que la interpretación epistémica de la distinción crítica fenómeno / cosa en sí no necesariamente niega ni desconoce las consecuencias metafísicas que se deducen de dicha distinción, sino simplemente destaca el hecho de que ambos conceptos no remiten a entidades ontológicamente diversas, sino a dos perspectivas bajo las que ha de ser considerado el objeto empírico (perspectivas que -como ha sido indicado- aluden a dos dimensiones ontológicas de dicho objeto ). En la sección siguiente, consideraremos en qué sentido cabe afirmar que la interpretación epistémica no reduce, sin embargo, las nociones de $f e$ nómeno y de cosa en sí a meras perspectivas (esto es, a conceptos heurísticos carentes de correlato real), sino que permite ser articulada, por el contrario, con una interpretación realista del fenómeno y de la cosa en sí, esto es: permite ser conciliada con una consideración de ambos como dimensiones realmente existentes del objeto empírico.

\section{LA EXISTENCIA DE LO EN SÍ COMO PRESUPUESTO BÁSICO DEL IDEALISMO TRASCENDENTAL}

En la sección previa hemos indicado que -desde la perspectiva adoptada por los partidarios de la lectura epistémica del IT- los conceptos de fenómeno y de cosa en sí remiten a un único objeto, a saber: aquel que es dado en la experiencia (ambos conceptos aluden, más precisamente, al modo en que dicho objeto ha de ser considerado en el marco de una reflexión trascendental acerca de las condiciones a priori de toda representación objetiva en general). Ahora bien, podría pensarse que la interpretación de ambos conceptos como nociones que aluden a perspectivas o modos de consideración conduce a la interpretación del fenómeno y la cosa en sí como términos puramente ficcionales que no designarían nada real (siendo, en rigor, el objeto empírico lo único real en sentido estricto). En otras palabras: podría pensarse que Kant no consideró a las cosas en sí como algo real sino que sus referencias a estas entidades incognoscibles se fundan en la pura necesidad teórica de establecer un correlato lógico-semántico del concepto de fenómeno. En tal sentido, podría arribarse a la conclusión de que la filosofía crítica no admite, en rigor, la existencia de 
entidades reales independientes del ámbito de la representación, sino que se limita a invocar la noción de cosa en sí a título de mero concepto ficcional (y ello por razones puramente doctrinales).

Por nuestra parte, consideramos que tal interpretación resulta inconsistente, principalmente debido a la existencia de numerosos pasajes que permiten constatar que Kant no consideró al fenómeno ni a la cosa en sí como entidades puramente ficcionales. ${ }^{16}$ En lo que respecta a la noción de fenómeno, el filósofo deja claro que la idealidad trascendental de los fenómenos sensibles no excluye (sino, por el contrario, se corresponde con) su realidad empírica.${ }^{17} \mathrm{Si}$ bien la cuestión relativa al status que ha de asignarse al fenómeno en el marco del Idealismo trascendental ha sido y continúa siendo ampliamente debatida entre los autores dedicados al estudio de la filosofía crítica, ${ }^{18}$ no es necesario que nos detengamos aquí a considerar esta cuestión, siendo nuestro principal objetivo examinar ciertos aspectos problemáticos referidos al concepto de cosa en sí. Nos limitaremos, así pues, a considerar brevemente los principales

16 Nuestra interpretación se opone, pues, a aquellas que podrían ser caracterizadas como interpretaciones idealistas de la cosa en sí, entendiendo por tales aquellas que establecen el carácter puramente ficcional de la noción de cosa en sí. Como ejemplo paradigmático de estas interpretaciones, vid. E. Schaper, «The Kantian Thing-in-Itself as a Philosophical Fiction», The Philosophical Quarterly, 16, 64 (1966), pp. 233-243. Los partidarios de tal interpretación idealista suelen destacar la función meramente limitativa que el concepto de cosa en sí desempeña -o debería desempeñar- en el marco de la filosofía crítica (J. Baumgartner, «On Kant’s Matter of Appearance», en G. Funke (de.), Akten des 4. Internationalen Kant-Kongresses. Berlin, Walter de Gryter, 1974, p. 267; N. Rescher, «On the status of Things in Themselves in Kant», Synthese, 27 (1981), pp. 296-297). Consideramos, sin embargo, que el reconocimiento de la indudable función limitativa que el concepto de cosa en sí desempeña en la doctrina crítica no impide reconocer que Kant consideró a las cosas en sí como entidades reales, y no como meros entes de razón. Más aún: si la cosa en sí establece el límite insuperable más allá del cual no es posible, para nosotros, conocimiento alguno, es precisamente en su carácter de entidad real, pues un límite meramente pensado no alcanzaría a constituir, en última instancia, un auténtico límite. El principio crítico que establece el carácter estrictamente fenoménico de todo conocimiento humano sólo tiene sentido - a nuestro juicio- si se admite la existencia efectiva de un dominio real, más allá del orden puramente subjetivo de nuestras representaciones.

$17 C f$. A 28/B 44. Así como la idealidad trascendental del tiempo y el espacio se articula con su realidad empírica, así también podemos afirmar que los fenómenos espacio-temporales son ideales en sentido trascendental, pero a la vez reales en sentido empírico.

18 Cf. G. Prauss, Erscheinung bei Kant. Ein Problem der Kritik der reinen Vernunft. Berlín, 1971. R. Lay, «Realität und Erscheinung», en G. Funke (ed.), Akten des 4. Internationalen Kant-Kongresses. Berlin: Walter de Gruyter, 1974, pp. 204-213; K. Ameriks, «Kant's Idealism on a Moderate Interpretation», en D. Schulting y J. Verburgt (eds.), Kant's idealism. New Interpretation of a Controversial Doctrine. Springer, 2010, pp. 29-53; R. Brandt, «Transzendentale Ästhetik §§ 1-3» en G. Mohr y M. Willascheck (eds.), Klassiker Auslegen. Immanuel Kant : Kritik der reinen Vernunft, Berlin: Akademie, 1998, pp. 81-106. 
pasajes que permiten dar sustento a lo que hemos caracterizado aquí como una interpretación realista de la cosa en sí, entendiendo por tal aquella que considera el concepto de cosa en sí como noción que alude a una entidad real o existente. ${ }^{19}$ Finalmente, haremos referencia a los aspectos conceptuales que permiten articular esta interpretación realista del concepto de cosa en sí con la interpretación epistemológica de la distinción fenómeno / cosa en sí a la que hemos aludido previamente.

En el Prólogo a la segunda edición de la Crítica de la razón pura, Kant señala que nuestro conocimiento a priori «sólo se dirige a fenómenos, mientras que deja de lado a la cosa en sí misma [como una cosa que es], por cierto, efectivamente real en sí [für sich wirklich], pero desconocida para nosotros» (B XX). En el $§ 13$ de Prolegómenos afirma:

Admito, ciertamente, que hay cuerpos fuera de nosotros, esto es, cosas que conocemos mediante las representaciones que nos produce su influjo sobre nuestra sensibilidad, aunque nos son completamente desconocidas en lo que respecta a cómo sean en sí mismas; cosas a las que damos el nombre de cuerpo, palabra que entonces significa solamente el fenómeno de aquel objeto desconocido para nosotros, pero no por ello menos real [aber nichs desto weniger wirklichen Gegenstandes] (Ak. IV, 289).

En el mismo parágrafo declara que el idealismo crítico no suprime -a diferencia del auténtico idealismo- «la existencia de la cosa que aparece, sino que solamente señala que no podemos, mediante los sentidos, conocer esta cosa tal como es en sí misma» [denn die Existenz des Dinges, was erscheint, wird dadurch nicht wie beim wirklichen Idealism aufgehoben, sondern nur gezeigt, dass wir es, wie es an sich selbst sei, durch Sinne gar nicht erkennen können] (Ak. IV, 289). Más adelante, en el \$32 del mismo texto, observa que «precisamente porque admite fenómenos, el entendimiento acepta también la existencia de cosas en sí mismas» [Der Verstand also, eben dadurch dass er Erscheinungen annimmt, gesteht auch das Dasein von Dingen an sich selbst zu] (Ak. IV, 315). En el \$26 de la Analítica de los conceptos, señala, en el mis-

19 Las interpretaciones subjetivistas y / o fenomenalistas del Idealismo Trascendental suelen oponerse, en términos generales, a las interpretaciones realistas, las cuales resultan, a su vez, de índole muy diversa, en la medida en que pueden ser incluidas, bajo este título general, tanto a las lecturas que enfatizan el realismo empírico implicado en el idealismo trascendental (cf. P. Abela, Kant's Empirical Realism. Clarendon Press, Oxford, 2002) como a aquellas lecturas que destacan, por el contrario, lo que cabría caracterizar como un realismo trascendente subyacente al idealismo crítico, aludiéndose aquí bajo la noción de realismo trascendente a la convicción kantiana respecto de la existencia de una realidad trascendente al orden de la representación, interpretación que se remonta al texto clásico de E. Adickes, Kant und das Ding an Sich. Berlin: Panverlag Rolf Heise, 1924. 
mo sentido, que «los fenómenos son sólo representaciones de cosas que están presentes sin ser conocidas en lo que respecta a lo que puedan ser en sí» [Allein Erscheinungen sind nur Vorstellungen von Dingen, die, nach dem, was sie an sich sein mögen, unerkannt da sind] (B 164).

El primero de los pasajes referidos destaca el límite insuperable que la doctrina crítica establece respecto del alcance del conocimiento humano, al restringir dicho cocimiento al ámbito fenoménico. En el marco de estas observaciones, el concepto de cosa en sí alude precisamente a aquello que, aún siendo real en sí, no puede sin embargo, ser aprehendido por nuestras facultades cognitivas, a causa de las condiciones formales que éstas imponen a las cosas en tanto las representan. ${ }^{20}$ Los dos pasajes correspondientes al $\$ 13$ de Prolegómenos remiten, en cambio, al modo en que ha de ser interpretado el Idealismo trascendental en tanto doctrina filosófica que -a diferencia del idealismo convencional-no pretende negar o cuestionar la existencia de los cuerpos externos. En relación con esta importante cuestión, Kant señala que las cosas que nos afectan (produciendo nuestras representaciones empíricas) no son percibidas tal como son en sí; y añade a continuación que, si bien a estas cosas externas percibidas las denominamos cuerpos, los llamados cuerpos no constituyen, en sentido estricto, sino objetos representacionales; en otros términos: constituyen representaciones de cosas que permanecen, en sí, desconocidas para nosotros. Esto no significa, sin embargo, que los cuerpos externos sean considerados aquí como algo realmente diverso de las entidades que los producen al afectarnos; consideramos, por el contrario, que lo que aquí pretende destacarse es que percibimos esas cosas reales que nos afectan sólo tal como las configuramos a partir de formas a priori inherentes a nuestra facultad de representación. Eso real que nos afecta - permaneciendo, sin embargo, absolutamente desconocido para nosotros- no constituye, así pues, una entidad ontológicamente diversa del objeto representado. En otras palabras: la cosa en sí es eso mismo que aparece ante nosotros, siendo percibido, sin embargo, únicamente bajo su dimensión o aspecto fenoménico. ${ }^{21}$

Finalmente, el pasaje correspondiente al §32 de Prolegómenos permite constatar que la correspondencia entre los conceptos de fenómeno y de cosa en sí no es puramente lógica (o semántica), sino que se trata de un correlación entre dos dimensiones ontológicas correspondientes a un objeto único: Kant no se limita allí, en efecto, a afirmar que la consideración de los objetos como fenómenos exige a la vez una consideración de los mismos como cosas en sí (es decir, no

20 Esto significa que precisamente las mismas condiciones formales a priori que hacen posible el conocimiento ( $y$, en general, la experiencia objetiva) como tales, son las que determinan asimismo los límites infranqueables de toda experiencia y conocimiento humanos.

21 Cf. supra, nota 13. 
se limita a establecer una correlación estrictamente lógico-semántica entre las nociones de fenómeno y de cosa en sí), sino que declara que, al admitirse la existencia del fenómeno, ha de admitirse asimismo la existencia de lo en sí, pues esta última se halla presupuesta en la existencia misma del fenómeno; y esto es así porque ambos modos de existencia se hallan igualmente comprendidos en la existencia del objeto empírico. Cabría afirmar, así, que dicho objeto existe como fenómeno (esto es: posee una existencia espacio-temporal en tanto objeto representado) y existe, a la vez, como algo en sí (i.e. posee una existencia independiente del sujeto de la representación). Podemos concluir, pues, que desde la perspectiva teórica asumida por la filosofía crítico-trascendental, los objetos empíricos son considerados como representaciones sensibles de cosas reales que nos afectan, sin que podamos percibirlas, sin embargo, tal como son en sí mismas (tal como señala Kant en el pasaje correspondiente al \$26 de la Analítica de los conceptos, al que hemos aludido previamente).

Los pasajes previamente referidos no sólo permiten advertir que la doctrina crítica no pretende en modo alguno cuestionar la existencia de entidades independientes con respecto al orden de la representación (es decir, no pretende poner en duda la existencia de cosas reales en sí), ${ }^{22}$ sino que permiten además constatar que la existencia de un orden trascendente al orden de la representación constituye, para el filósofo crítico, un supuesto o punto de partida básico e inconmovible de la investigación trascendental, supuesto que no parece requerir de demostración alguna. ${ }^{23}$ Que no podamos asignar un valor de conocimiento

22 Así afirma Kant, en el $\$ 13$ de Prolegómenos, y a fin de evitar la identificación de su doctrina con el «idealismo en sentido tradicional»: «Pues el que yo haya dado a esta teoría mía el nombre de idealismo trascendental no autoriza a nadie a confundir este nombre con el idealismo empírico de Descartes [...], ni con el idealismo místico de Berkeley [...]. Pues este idealismo que yo he llamado así no se refería a la existencia de las cosas (la duda acerca de la cual constituye propiamente el idealismo en el sentido tradicional). Pues nunca se me ocurrió ponerla en duda; sino que se refiere solamente a la representación sensible de las cosas, a la cual pertenecen, por sobre todo, el espacio y el tiempo» (Ak. IV, 293).

23 Coincidimos con Adickes cuando señala que Kant simplemente asume dicha existencia, y que este supuesto opera como un principio básico de la doctrina del idealismo trascendental, la cual en modo alguno pretende cuestionar la existencia de una realidad trascendente al sujeto, sino que únicamente rechaza su cognoscibilidad: «Nach meiner Überzeugung ist für Kant in seiner ganzen kritischen Zeit die transsubjektive Existenz einer Vielheit von Dingen an sich, die unser Ich affizieren, eine nie bezweifelte, absolute Selbstverständlichkeit gewesen» (E. Adickes, op . cit., p. 4). Así concluye el autor: «Es liegt eben, was die Existenz der Dinge an sich betrifft, für Kant überhaupt kein problem vor. Sie ist eine unbewiesene Prämisse, von der er ausgeht, als sei sie so sicher wie der sicherst bewiesene Gundsatz. [...] In unserem Fall beweisen sie [...], wie stark die realistische Tendenz in Kant war, und wie fern ihm deshalb jeder extreme Idealismus liegen musste» (E. Adickes, op. cit., p. 9). Véase asimismo: E. Adickes, op. cit., pp. 156ss.; M, 
a esta suposición básica, no afecta al hecho de que tal suposición constituya el punto de partida inicial del análisis trascendental, en tanto investigación filosófica que procura elucidar las condiciones a priori que hacen posible la representación objetiva de esas entidades reales que nos afectan ${ }^{24}$. Si a partir de tal investigación Kant concluye que -precisamente a causa de dichas condiciones a priori-no podemos conocer las cosas tal como son en sí mismas (de manera tal que la existencia de lo real en sí no puede ser conocida, sino sólo puede ser pensada), ello no implica, sin embargo, que la existencia de las cosas en sí se reduzca a su ser pensadas, ${ }^{25}$ sino sólo indica que nosotros no podemos conocer objetivamente dicha existencia (dado que las formas a priori inherentes a nuestra facultad de representación -espacio, tiempo y categorías- sólo tienen un uso legítimo empírico, esto es, un uso referido a la determinación del objeto como fenómeno).

Ahora bien, el punto decisivo para nuestro análisis es que eso real en sí que nos afecta, permaneciendo, no obstante, absolutamente desconocido para nosotros, no ha de ser considerado como algo diverso del objeto dado en la experiencia, sino, antes bien, ha de ser asumido como un aspecto o dimensión (para nosotros incognoscible) de tal objeto. De este modo, la interpretación realista de la cosa en sí no conduce a una interpretación ontológica de la distinción fenómeno / cosa en sí, siendo aquélla compatible, por el contrario, con una interpretación epistémica de tal distinción.

Caimi, «About the Argumentative Structure of the Transcendental Aesthetic», Studi Kantiani, 9 (1996), pp. 42-43.

24 El llamado problema de la afección ha sido intensamente debatido en el marco de la discusión entre los partidarios de las lecturas ontológicas y los defensores de la interpretación epistémica del IT. Si bien no podemos detenernos aquí a considerar detalladamente la cuestión, cabe señalar que, desde una lectura epistémica, la caracterización kantiana de las entidades que afectan a la sensibilidad como cosas en sí no implica la postulación de entidades suprasensibles ontológicamente diversas de los fenómenos sensibles, sino que dicha caracterización sólo indica, en última instancia, de qué manera ha de ser pensada o concebida la entidad afectante en el marco de la investigación trascendental ( $c f$. H. Allison, Kant's Transcendental Idealism, p. 72). Para un análisis del problema de la afección, véase: M, Caimi, «About the Argumentative Structure of the Transcendental Aesthetic», Studi Kantiani, 9 (1996), pp. 42-43; G. Buchdahl, «A Key to the Problem of Affection», en G. Funke (ed.), Akten des Siebenten Internationalen Kant-Kongresses. Berlin: Walter De Gruyter, 1991, pp. 73-90; G. Prauss, Kant und das Problem der Dinge an sich. Bonn: Bouvier Verlag, 1989, pp. 192-207, I. Beade, «Acerca de la cosa en sí como causa de la afección sensible», Signos Filosóficos, XII, 23 (2010), pp. 9-37; N. Rescher, «Noumenal Causality», en L. W. Beck (ed.), Kant's Theory of Knowledge. Boston: Reidel Publishing Company, 1974, pp. 178ss.

25 En tal sentido observa Rábade Romeo que «una cosa es que la cosa-en-sí sólo pueda ser pensada y otra que su en-sidad se reduzca a ese ser pensada» (S. Rábade Romeo, Kant. Problemas gnoseológicos de la Crítica de la Razón pura. Madrid: Gredos, 1969, p. 97). 


\section{AlgunAs CONSIDERACIONES FINALES}

En la sección precedente hemos procurado exponer los principios básicos sobre los que se sustenta lo que ha sido caracterizado aquí como una interpretación realista de la cosa en sí (entendiendo por tal interpretación aquella que considera a la cosa en sí como entidad real o existente, no obstante su carácter absolutamente incognoscible, y pese a las complejas dificultades implicadas en la aplicación de las categorías de realidad y existencia a la cosa en $s i^{26}$ ). Hemos procurado asimismo aclarar en qué sentido esta interpretación realista de la cosa en sí resulta compatible con la interpretación epistemológica de la distinción fenómeno / cosa en sí, cuyos lineamientos básicos han sido expuestos en la sección primera de este escrito.

Insistir acerca de la vinculación entre ambas interpretaciones resulta, desde luego, fundamental, atendiendo a los objetivos aquí formulados, ya que -como hemos indicado- podría asumirse que una interpretación realista de la cosa en sí ha de conducir necesariamente a una interpretación ontológica de la distinción fenómeno / cosa en sí (esto es: podría pensarse que la consideración de la cosa en sí como entidad real implica ipso facto la concepción de ésta como entidad realmente diversa del fenómeno sensible). Nuestras observaciones precedentes concluir, sin embargo, que este no es el caso: no se da, en efecto, una correlación necesaria entre la concepción de la cosa en sí como entidad real y la interpretación ontológica de la distinción fenómeno / cosa en sí, pues -como hemos intentado mostrar- la cosa en sí es considerada, en el marco de la doctrina crítica, como algo realmente existente, sin que ello implique, no obstante, que ella constituya una entidad realmente diversa del objeto fenoménico. Hemos sugerido que tanto el concepto de cosa en sí como el concepto de fenómeno hacen referencia a dimensiones reales de un objeto único: el objeto empírico (en el caso de la noción de fenómeno, se trata de un concepto que permite considerar dicho objeto tal como aparece ante el sujeto de la representación; en el caso del concepto de cosa en sí, se trata en cambio de la consideración de dicho objeto independientemente de toda relación con el sujeto). Ahora bien, el punto decisivo es que ambas dimensiones del objeto empírico no han de ser consideradas como dos modalidades diversas e independientes de existencia, sino antes bien como dos dimensiones ontológicas igualmente constitutivas de un objeto único existente. Dicho objeto - cabría afirmar-existe como fenómeno y existe como cosa en sí, siendo ambas dimensiones respectivamente relativas a las dos perspectivas bajo las cuales es considerado el objeto empírico en el marco del análisis trascendental. De este modo, la interpretación epistémica de la distinción fenómeno / cosa en sí permite ser articulada con una interpretación

26 Dado que no es posible ofrecer aquí un análisis detallado de tales dificultades, remitimos al lector a nuestros trabajos previos sobre la cuestión (cf. supra, nota 6). 
realista del fenómeno y de la cosa en sí (constituyendo el fenómeno una entidad real en sentido empírico, y la cosa en sí una entidad real en sentido trascendente). La interpretación epistémica no implica, en síntesis, la reducción de la cosa en sí a un mero concepto, sino que permite, por el contrario, garantizar su realidad, pues -reiteramos- al no constituir aquella algo realmente diverso del objeto empírico, en la existencia de éste se halla comprendida ipso facto la existencia de aquella (lo mismo cabría afirmar respecto del fenómeno).

Quizás la consecuencia más relevante de la interpretación epistémica de la distinción fenómeno / cosa en sí es que permite advertir que tal distinción no compromete al Idealismo trascendental con un dualismo metafísico (que resultaría dogmático y, por tanto, contrario al espíritu crítico de dicha doctrina). Por su parte, la interpretación realista de la cosa en sí permite reconocer que -a diferencia de lo que sostienen los partidarios de las lecturas fenomenalistasdicha doctrina no reduce lo real a un mero estado de conciencia (ni el fenómeno es considerado, en dicho marco, como una pura representación puramente mental, ni la cosa en sí es concebida como un mero concepto heurístico sin correlato real alguno; de tal modo que la realidad-empírica- del fenómeno como la realidad -trascendente- de la cosa en sí resultan allí, por el contrario, igualmente preservadas; si bien sólo la existencia del fenómeno puede ser objetivamente conocida, mientras que la existencia de lo en sí no puede ser, en rigor conocida, sino que ha de ser asumida a modo de supuesto básico, tal como hemos sugerido en la segunda sección). Consideramos, por lo dicho, que ambas interpretaciones revelan aspectos decisivos para la interpretación del IT en su conjunto, y no es sino a partir de su articulación que logran dar cuenta del sentido específico que la noción de cosa en sí desempeña en el desarrollo de la investigación trascendental, en tanto concepto filosófico que no sólo permite establecer el límite insuperable de todo conocimiento humano, sino que permite asimismo preservar los presupuestos realistas básicos sobre los que se sustenta el idealismo crítico, en cuyo marco no pretende cuestionarse la existencia de cosas reales, sino sólo procura hacerse explícito el carácter puramente fenoménico de los objetos dados en la experiencia. ${ }^{27}$

\section{REFERENCIAS BIBLIOGRÁFICAS}

ABELA, P., Kant's Empirical Realism. Oxford: Clarendon Press, 2002.

27 Cf. supra, nota 22. 
ADICKES, E., Kant und das Ding an Sich. Berlin: Panverlag Rolf Heise, 1924.

ALLAIS, L., «Kant's one world: Interpreting Transcendental Idealism», British Journal for the History of Philosophy, 12, 4 (2004), pp. 655-684.

ALLISON, H., «Kant's Refutation of Realism», Dialéctica, 30, 2/3 (1976,) pp. 287288.

ALLISON, H., Idealism and Freedom. Essays on Kant's Theoretical and Practical Philosophy. Cambridge: Cambridge University Press, 1996.

ALLISON, H., Kant's Transcendental Idealism. New Haven: Yale University Press, 2004

AMERIKS, A., Interpreting Kant's Critiques. Oxford: Clarendon Press, 2003.

AMERIKS, K., «Kant's Idealism on a Moderate Interpretation», en D. Schulting y J. Verburgt (eds.), Kant's idealism. New Interpretation of a Controversial Doctrine. Springer, 2010, pp. 29-53.

BAUMGARTNER, J., «On Kant's Matter of Appearance», en G. Funke (comp.), Akten des 4. Internationalen Kant Kongresses. Berlin, Walter de Gryter, 1974, pp. 265-269.

BEADE, I., «Consideraciones acerca del concepto kantiano de objeto trascendental», Tópicos, 22 (2009), pp. 85-120;

BEADE, I., «Acerca de la cosa en sí como causa de la afección sensible», Signos Filosóficos, XII, 23 (2010), pp. 9-37.

BEADE, I., «Acerca del concepto kantiano de fenómeno y su relación con el concepto crítico de cosa en sí», en D. López, M. Yuan y C. Lammertyn (eds.), Experiencia y concepto. Intensidades clásicas y tensiones contemporáneas. Santa Fe: Ediciones de la Universidad Nacional del Litoral, 2011, pp. 254-260.

BEADE, I., «La cosa en sí y su rol en la constitución de la objetividad. Una lectura crítica de la interpretación de Ch. Onof», en M. Damiani (de.), Actas del I Simposio de Filosofía Moderna, Rosario: Centro de Estudios de Filosofía Moderna, 2011, (en prensa).

BEADE, I., «Realidad y perspectiva. Consideraciones acerca del concepto de cosa en sí en el marco de la Gnoseología crítica», en D. López y S. Di Sanza (eds.), El idealismo alemán. Autores y textos. Santa Fe: Ediciones de la Universidad Nacional del Litoral, (en prensa).

BRANDT, R., «Transzendentale Ästhetik §§ 1-3» en G. Mohr y M. Willascheck (eds.), Klassiker Auslegen. Immanuel Kant:Kritik der reinen Vernunft, Berlin: Akademie, 1998, pp. 81-106.

BUCHDAHL, G. «A Key to the Problem of Affection», en G. Funke (ed.), Akten des Siebenten Internationalen Kant-Kongresses. Berlin: Walter De Gruyter, 1991, pp. 73-90.

CAIMI, M. «About the Argumentative Structure of the Transcendental Aesthetic», Studi Kantiani, 9 (1996), pp. 27-46.

CHENET, F. X., «Que sont donc l'espace et le temps? Les hypothèses considérées par Kant et la lancinante objection de la «'troisième possibilité»» Kant Studien, 84 (1993), pp. 129-153.

FALKENSTEIN, L. «Kant's Argument for the Non-Spatiotemporality of Things in Themselves», Kant Studien, 80 (1989), pp. 265-283; 
HEIDEMANN, D., «Appearance,Thing-in-Iself, and the Problem of the Skeptical Hypothesis», en D. Schulting y J. Verburgt, (eds.), Kant's Idealism. New Interpretations of a Controversial Doctrine. Springer, 2010, pp. 195-210.

HUENEMANN, Ch., "A Note on the Argument fot the Non-Spatiotemporality of Things in Themselves. A response to Lorne Falkenstein», Kant Studien, 83 (1993), pp. 381-383.

KANT, I., Kant's gesammelte Schriften, Berlin et alia: Deutschen Akademie der Wissenschaften, 1902ss.

KANT, I., Crítica de la razón pura, tr. Mario Caimi. Buenos Aires: Colihue, 2007.

KANT, I., Prolegómenos a toda metafísica que haya de poder presentarse como ciencia, tr. M. Caimi. Madrid: Istmo, 1999.

LAY, R., «Realität und Erscheinung», en G. Funke (ed.), Akten des 4. Internationalen Kant Kongresses. Berlin: Walter de Gruyter, 1974, pp. 204-213.

ONOF, Ch., «Thinking the In-itself, and Its Relation to Appearances», en D. Schulting y J. Verburgt (eds.), Kant's Idealism. New Interpretation of a Controversial Doctrine. Springer, 2010, pp. 211-235.

PATON, H. J., Kant's Metaphysic of Experience. A Commentary on the First Half of the Kritik der reinen Vernunft Londres: Georg Allen \&. Unwin, 1970, vol. I, p. 422).

PRAUSS, G., Erscheinung bei Kant. Ein Problem der Kritik der reinen Vernunft. Berlin: Walter De Gruyter, 1971.

PRAUSS, G., Kant und das Problem der Dinge an sich, Bonn: Bouvier Verlag, 1989.

RÁBADE ROMEO, S., Kant. Problemas gnoseológicos de la Crítica de la Razón pura. Madrid: Gredos, 1969.

RESCHER, N., «Noumenal Causality», en L. W. Beck (ed.), Kant's Theory of Knowledge. Boston: Reidel Publishing Company, 1974, pp. 174-183.

RESCHER, N., «On the status of Things in Themselves in Kant», Synthese, 27 (1981), pp. 289-299.

SCHAPER, E., «The Kantian Thing-in-Itself as a Philosophical Fiction», The Philosophical Quarterly, 16, 64 (1966), pp. 233-243.

SCHULTING, D., «Kant's Idealism: The Current Debate», en D. Schulting y J. Verburgt (eds.), Kant's Idealism. New Interpretations of a Controversial Doctrine. Springer, 2010, pp. 1-35.

WESTPHAL, M., «In Defense of the Thing in Itself», Kant Studien, 59 (1968), pp. 118-141.

Ileana Paola Beade es Profesora de la Cátedra de Filosofía Moderna de la Facultad de Humanidades y Artes de la Universidad Nacional de Rosario (Argentina) e Investigadora Asistente del Consejo Nacional de Investigaciones Científicas y Técnicas (CONICET, Argentina).

Publicaciones recientes:

BEADE, I., «Acerca del estatus epistemológico de las observaciones kantianas referidas a la existencia de las cosas en sí». Revista de Filosofía (Universidad Complutense de Madrid) 
35.2 (2010), pp. 43-57.

BEADE, I., «Libertad y Naturaleza en la Filosofía kantiana de la Historia». Daimon 54 (2011), pp. 25-44 (ISSN: 1130-0507).

\section{Líneas de investigación}

Problemas específicos de la epistemología kantiana, tales como la distinción fenómeno / cosa en sí, el llamado problema de la afección trascendental (vinculado a la caracterización kantiana de la cosa en sí como entidad que afecta a la sensibilidad, dando así origen a nuestras representaciones empíricas), y las diversas proyecciones prácticas de dicha distinción.

Filosofía político-jurídica kantiana y filosofía kantiana de la historia.

Dirección electrónica: ileanabeade@yahoo.com 
